\title{
Acute Pulmonary Coccidioidomycosis and Review of Published Cases with Lung Involvement in Korea
}

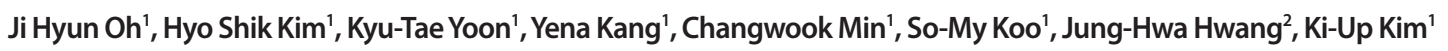 \\ Departments of 'Internal Medicine and ' Radiology, Soonchunhyang University Hospital, Soonchunhyang University College of Medicine, Seoul, Korea
}

\begin{abstract}
Coccidioidomycosis is a fungal infection caused by Coccidioides immitis. The endemic area is mostly south-western United States. As increasing in overseas travel to endemic areas, the incidence rate has been recently increased in non-endemic areas. The diagnosis may be delayed in non-endemic area. It is important to elicit traveling histories and to differentiate lung consolidation with eosinophilia, for timely diagnosis of coccidioidomycosis. Recently, we experienced a case with pulmonary coccidioidomycosis in a Korean American who visited Korea showed consolidation in right lower lobe on chest X-ray and prolonged eosinophilia. In the case, a confirmatory diagnostic method was percutaneous transthoracic needle biopsy of lung. We report acute pulomonary coccidioidomycosis case and review previous published reports with pulmonary manifestation in Korea.
\end{abstract}

Keywords: Pulmonary coccidioidomycosis; Endemic diseases; Pulmonary fungal infection

\section{INTRODUCTION}

Coccidioidomycosis is an endemic fungal infection of the southwestern United States and parts of Mexico and Central and South America. Coccidioides species (Coccidioides immitis or Coccidioides posadasii) are causative pathogens by inhaling the spores [1]. As increasing in overseas travel to endemic areas, the incidence rate of coccidioidomycosis has been recently increased in non-endemic areas. In Korea, sixteen cases of coccidioidomycosis have been reported since 1976, and the incidence of infection has increased in the last 10 years [2]. But the diagnosis of coccidioidomycosis is often delayed because the infection is not initially considered.

Coccidioidomycosis has been reported to involve almost all organ systems, however, the most common clinical presentation is lung involvement [3]. Most of them are self-limited, and only a few who has immunocompromised host develop disseminated disease [1].

The diagnosis of acute pulmonary coccidioidomycosis can be made by direct microscopic examination of lower respiratory tract specimens for Coccidioides spherules and culture of such specimens. Serologic tests are also used frequently. Twenty-five percent to $30 \%$ of patients showed eosinophilia in peripheral blood [4]. Coccidioidomycosis must be considered one of the differential diagnosis of pulmonary infiltrates with peripheral eosinophilia, especially if patient has lived in or visited endemic area.

Here, we report a case of acute pulmonary coccidioidomycosis in a Korean American who has traveled in Arizona, USA. We also reviewed previous published coccidioidomycosis cases with lung involvement in Korea.

\section{CASE REPORT}

A 50-year-old male, a Korean American, was referred due to eosinophilia with lung consolidation. He had been diagnosed pneumonia 5 days ago based on fever, chill, cough, myalgia, and abnormal consolidation on chest radiography. After intravenous antibiotics and conservative care, he had recovered from systemic symptoms but remained consolidation on chest radiography and eosinophilia in peripheral blood. The patient was ex-smoker and lived near Los Angeles in United States. He had diabetes and had been taken oral medicine for 5 years. He was engaged in transportation. 
He came to Korea 12 days ago for vacation.

On physical examination, the patient was conscious, oriented, and afebrile. His blood pressure was 130/90 $\mathrm{mm} \mathrm{Hg}$ and pulse rate was 100/min. During fever, maculopapular rashes had been developed on trunk and antecubital area but almost faded out in our clinic. Physical examination of chest was not different from pneumonia including rales, dullness on percussion, and increased tactile fremitus. Other systemic examination was unrevealed. We checked his chest radiography; it showed airspace consolidation with air-bronchogram and ground glass appearance (GGA) on right lower lung field (Fig. 1).

Complete blood count showed an elevated leukocyte count $14,800 / \mu \mathrm{L}$ (reference range, 4,000 to $10,000 / \mu \mathrm{L}$ ) and elevated eosinophils $2,800 / \mu \mathrm{L}$ (19.0\%). Other laboratory tests including chemistries and serologic tests were within normal ranges. But we prescribed oral antibiotics and supportive medicine for a week. Nevertheless the eosinophilia and lesion on chest radiography remained
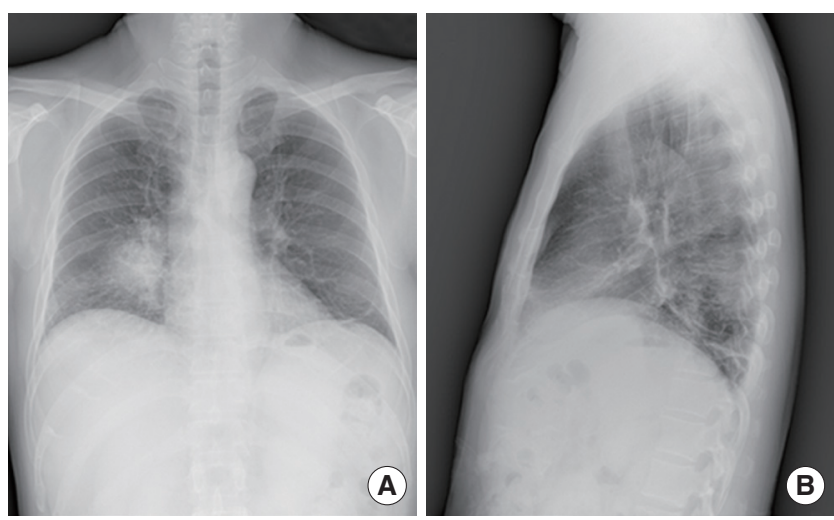

Fig. 1. (A) Chest PA at initial visit. It showed airspace consolidation with airbronchogram and ground glass appearance on right lower lung field. (B) Patchy consolidation was in retrocardiac area at left lateral. PA, posterial-anterial. consistently. He was taken computed tomography of chest, and it showed airspace consolidation with peribronchial GGA in right lower lobe associated with interstitial septal thickening and multiple poorly defined noncalcified centrilobular micronodules on both lungs (Fig. 2).

The procedure to confirm was needed, so percutaneous transthoracic needle biopsy (PTNB) was performed successfully at consolidated area. Pathologic report by hematoxylin eosin stain showed organized tissue with caseated area (Fig. 3A), accompanied by multinucleated giant cells and eosinophils, and we also found round eosin stained endospores which look like spherules (Fig. 3B). Dark colored spherules were scattered on Gomori's methenamine silver stain (Fig. 3C). Well capsulated red round ring was detected by Periodic acid Schiff stain (Fig. 3D).

Cultures for ordinary bacteria and acid fast bacilli were negative, but culture for fungus was white cottony molds at plate (Fig. 4A). Microscopic examination showed multiple arthroconidia which might be used to identify Coccidioides organism (Fig. 4B).

Final diagnosis was a pulmonary coccidioidomycosis. The patient was started treatment with itraconazole $400 \mathrm{mg} /$ day.

\section{DISCUSSION}

Major endemic regions of coccidioidomycosis are southern Arizona, central or other areas of California, southern New Mexico, and west of Texas [1]. In non-endemic area, diagnosis of coccidioidomycosis may be delayed or missed because of a low index of suspicion. According to a study in the United States in 2001, the median diagnosis were made 3 months after symptoms developed (range, 4 weeks \pm 14 months) [5].

Although coccidioidomycosis is not transmitted from person to
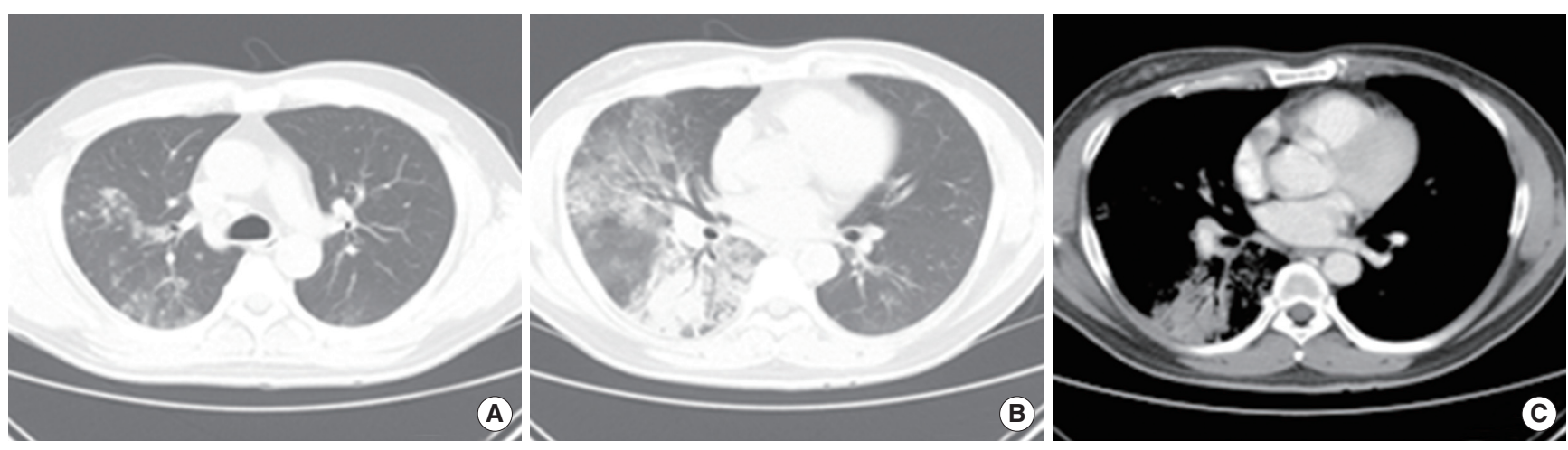

Fig. 2. Chest computed tomography shows (A) multiple poorly defined centrilobular micronodules and (B, C) consolidation with ground glass attenuation in right lower lobe with interlobular septal thickening, peribronchovascular ground glass appearances. 

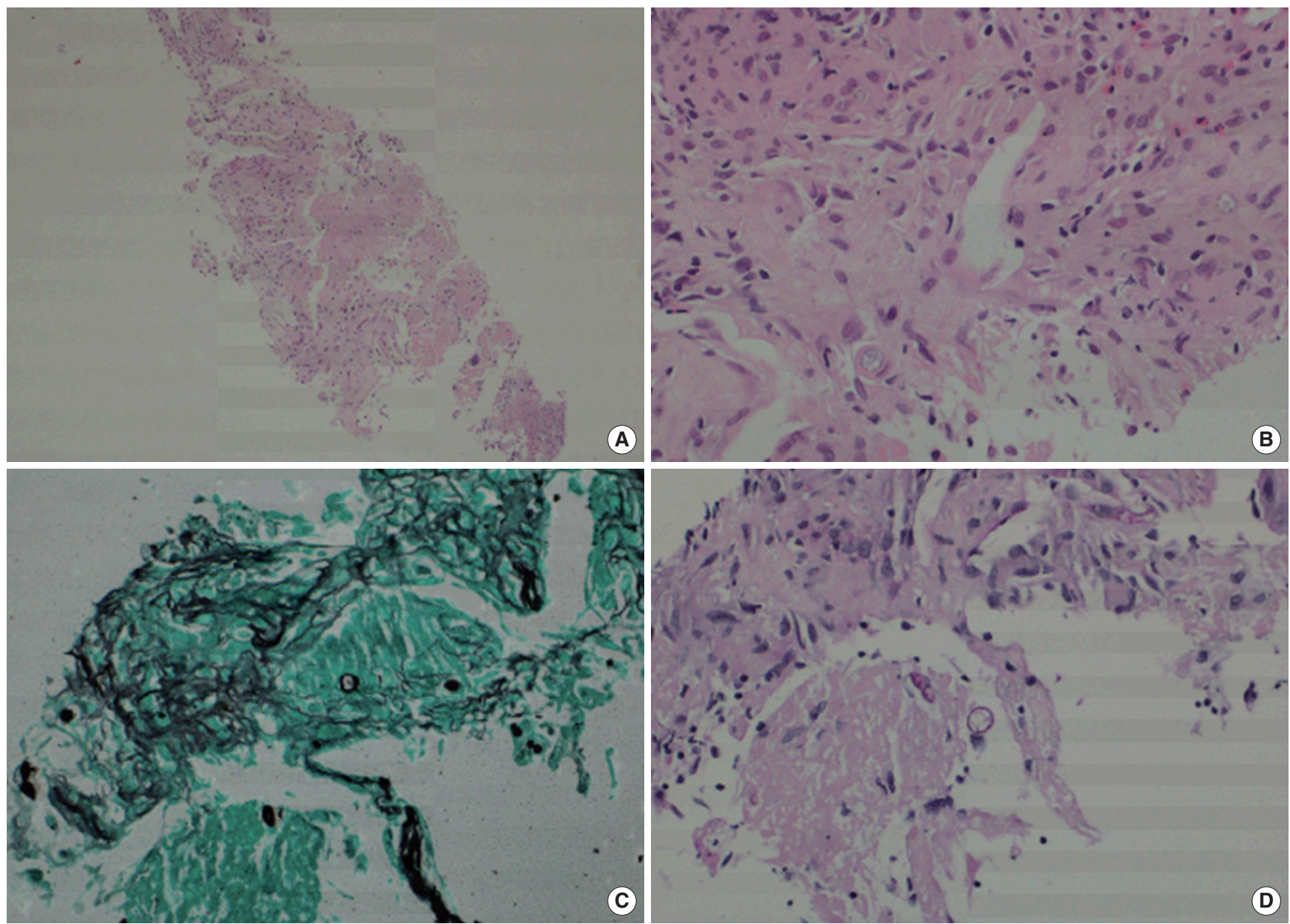

Fig. 3. Pathologic finding in lung biopsy from right lower lobe from the patient. (A) Alveolar structure with focal caseous necrosis (H\&E, $\times 40)$. (B) Eosinophilic infiltration and round eosin stained endospores looks like spherules (H\&E, $\times 100)$. (C) Dark colored spherules are scattered (Gomori's methenamine silver stain, $\times 100)$. (D) Well capsulated round ring which are stained red, are detected (Periodic acid Schiff stain, $\times 100$ ).
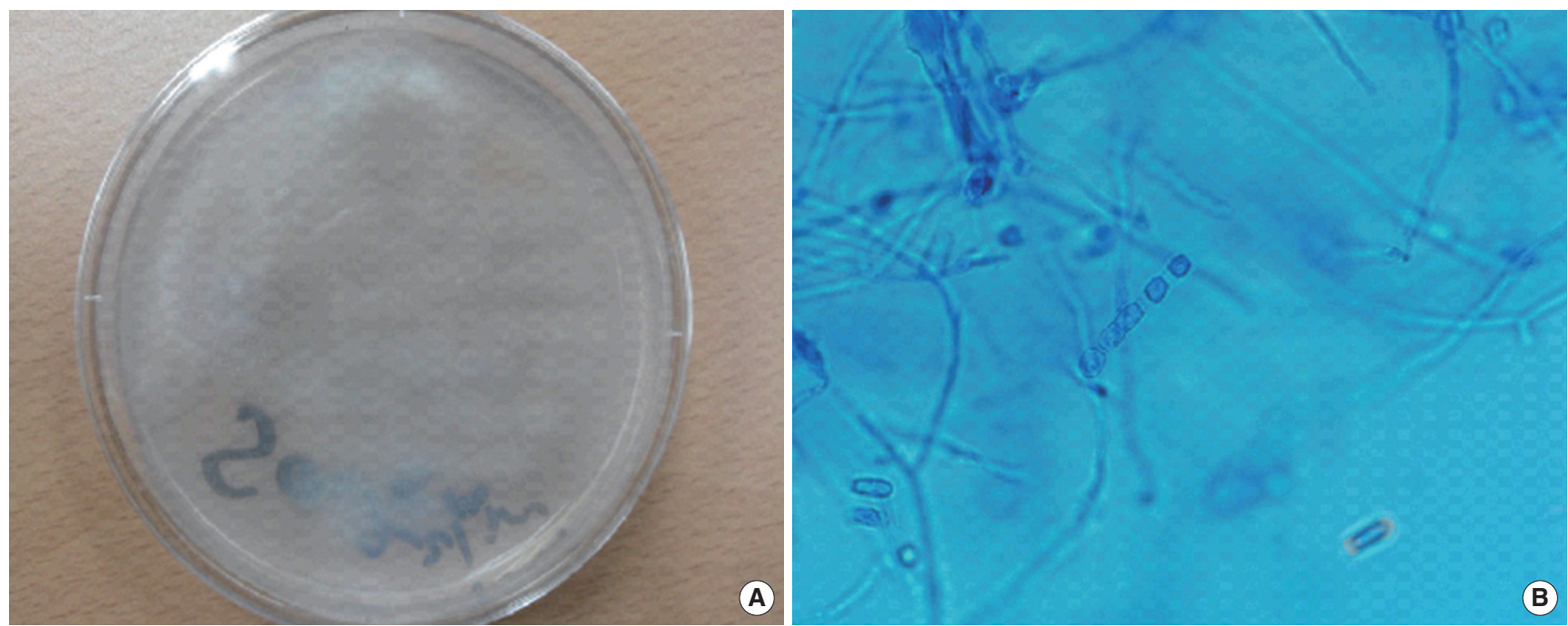

Fig. 4. Microbiologic finding in fungal culture with lung aspiration tissue. (A) White cottony molds at plate cultured during 2 weeks in Sabouraud dextrose agar. (B) Lactophenol cotton blue stain $(\times 1,000)$. 
person, infections are acquired through inhalation of arthroconidia, a type of fungal spore typically produced by segmentation of pre-existing fungal hyphae. After inhalation, the arthroconidia deposit in the bronchioles and develop into spherules with increasing numbers of endospores. When the spherules rupture, endospores can develop new spherules and extend infection [6]. Therefore, rapid and correct diagnosis is needed not only to treat of host but also to protect laboratory staff from work related exposure to this easily aerosolable infectious agent.

Approximately $60 \%$ of exposed subjects are asymptomatic and self-limited. Less than $5 \%$ of infected subjects have been progressed to pulmonary or extrapulmonary dissemination. Spread to extrapulmonary organ tends to occur more commonly in pregnant women, infants, non-Caucasians and immunocompromised hosts [6].

Symptomatic pulmonary coccidioidomycosis appears variously ranging from mild respiratory symptoms like flu and pneumonia to acute respiratory distress syndrome within a few weeks after exposure. In our patient, he drived coast to coast in America, lived in endemic area, and suffered from diabetes. He had enough chances of exposure. We thought he had been exposed recently. His symptoms after acute exposure were developed during an airplane flight.

In some cases, the area of pneumonia remains nodule or cavity forms, which can be seen asymptomatic, but it is difficult to discriminate from malignancies because of morphological similarity [7]. Therefore, in many cases, invasive biopsy procedure was performed. The reported coccidioidomycosis were 16 cases in Korea, and 9 of them were involved chest lesion, which was infiltrations, nodules, and mediastinal lymph nodes (Table 1).

Incidence of pulmonary coccidioidomycosis has increased in the last decade in Korea. The pulmonary involvement of coccidioidomycosis was 9 cases, and 3 of them were acute symptomatic disease (Table 1). Most of their chief complaint was fever, and radiologic findings of lung were nodules, consolidation, and effusion [8-16].

It is not possible to check serologic test rapidly in Korea. If atypical pneumonia is suspected, eosinophilia may be an important clue in the differential diagnosis of fungal infection. More than half of pulmonary coccidioidomycosis has eosinophilia in blood and bronchoalveolar lavage, and eosinophila is an important clue in non-endemic area [4]. Our patient also had eosinophilia in blood and tissue.

If clinical symptoms and chest radiographic findings persist and progress more severe, patients was immunocompromised with in-

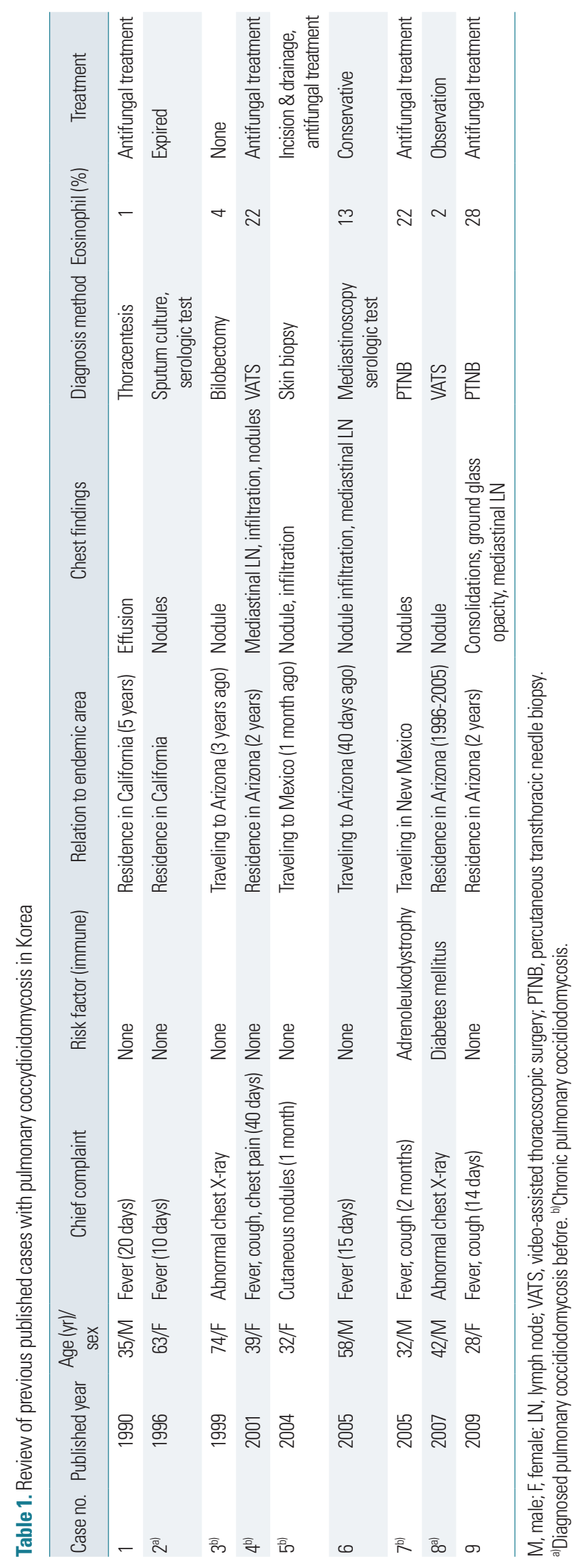


creased risk of dissemination, and anti-fungal treatment is recommended. Commonly prescribed therapies include amphotericin B, itraconazole, fluconazole, and ketoconazole. The duration of treatment ranges from three to six months, but this may be longer, especially in immunocompromised patients [1]. Although our patient had proper treatments, he was transferred with unresolved chest lesion to go back to US.

This is a typical acute pulmonary coccidioidomycosis, but in non-endemic area, the disease may have difficulty in diagnosis. Important clues of suspecting disease were history of living in the endemic area, eosinophilia at peripheral blood, and lung biopsy findings. So coccidioidomycosis must be taken into account in the differential diagnosis of pneumonia in case the patient is resistant to standard medication and has history of oversea travel or residence.

\section{REFERENCES}

1. Galgiani JN, Ampel NM, Blair JE, Catanzaro A, Johnson RH, Stevens DA, et al. Coccidioidomycosis. Clin Infect Dis 2005;41:1217-23.

2. Yoon HJ, Choi HY, Kim YK, Song YJ, Ki M. Prevalence of fungal infections using National Health Insurance data from 2009-2013, South Korea. Epidemiol Health 2014;36:e2014017.

3. Thompson GR 3rd. Pulmonary coccidioidomycosis. Semin Respir Crit Care Med 2011;32:754-63.

4. Malo J, Luraschi-Monjagatta C, Wolk DM, Thompson R, Hage CA, Knox KS. Update on the diagnosis of pulmonary coccidioidomycosis.
Ann Am Thorac Soc 2014;11:243-53.

5. Desai SA, Minai OA, Gordon SM, O’Neil B, Wiedemann HP, Arroliga AC. Coccidioidomycosis in non-endemic areas: a case series. Respir Med 2001;95:305-9.

6. Stevens DA. Coccidioidomycosis. N Engl J Med 1995;332:1077-82.

7. Spinello IM, Munoz A, Johnson RH. Pulmonary coccidioidomycosis. Semin Respir Crit Care Med 2008;29:166-73.

8. Kwak JH, Koo GW, Chung SJ, Park DW, Kwak HJ, Moon JY, et al. A case of significant endobronchial injury due to recurrent iron pill aspiration. Tuberc Respir Dis (Seoul) 2015;78:440-4.

9. Woo JH, Lee JS, Lee DW, Jin SY, Kim DW, Lee WG. A case of disseminated coccidioidomycosis--autopsy report. J Korean Med Sci 1996;11:258-64.

10. Yang HS, Lee J, Lim CM, Lee SD, Koh Y, Kim WS, et al. A case of coccidioidomycosis manifested as solitary pulmonary nodule. Tuberc Respir Dis 1999;46:266-72.

11. Shin JS, Lee IS, Shin C, Kim A. Pulmonary coccidioidomycosis diagnosed in an immigrant. Tuberc Respir Dis 2001;51:448-52.

12. Park DJ, Jang YH, Lee SJ, Na GY, Kim DW, Kim SW. A case of coccidioidomycosis showing cutaneous and pulmonary nodules. Korean J Med Mycol 2004;9:190-5.

13. Sinn DH, Kwon YS, Choi SY, Chang WI, Park MK, Han J, et al. A case of pulmonary coccidioidomycosis mimicking lung cancer. Korean J Med 2005;69:419-23.

14. Kim JH, Hur GY, Jung KH, Jung HC, Park DW, Lee SY, et al. Disseminated coccidioidomycosis presenting with miliary nodules. Tuberc Respir Dis 2006;60:97-101.

15. Lee JE, Kim HJ, Cho YJ, Joh JS, Han SK, Shim YS, et al. A case of pulmonary coccidioidomycosis that was misdiagnosed as tuberculoma and a review of the reported cases in Korea. Korean J Med 2007;72(Suppl 2): 206-10.

16. Kim SW, Oh JY, Kim EJ, Park GM. Pulmonary coccidioidomycosis in immunocompetent patient. Tuberc Respir Dis 2009;66:220-4. 